\title{
Cosmic Strings in the Abelian Higgs Model with Conformal Coupling to Gravity
}

\author{
Y. Verbin ${ }^{\star}$ \\ Department of Natural Sciences, \\ The Open University of Israel, P.O.B. 39328, Tel Aviv 61392, Israel
}

\begin{abstract}
Cosmic string solutions of the abelian Higgs model with conformal coupling to gravity are shown to exist. The main characteristics of the solutions are presented and the differences with respect to the minimally coupled case are studied. An important difference is the absence of Bogomolnyi cosmic string solutions for conformal coupling. Several new features of the abelian Higgs cosmic strings of both types are discussed. The most interesting is perhaps a relation between the angular deficit and the central magnetic field which is bounded by a critical value.

PACS numbers: 11.27. + d, 04.20.Jb, 04.40.Nr
\end{abstract}

\section{Introduction}

Cosmic strings [1, 2] were introduced into cosmology by Kibble [3], Zel'dovich [4] and Vilenkin [5] as (linear) topological defects which may have been formed during phase transitions in the early universe. Cosmic strings are considered as possible sources for density perturbations and hence for structure formation in the universe.

The simplest geometry of a cosmic string is that of a straight infinite one, which may represent anyhow a generic cosmic string from close up. The straight infinite (and static) string is therefore a source of a static and cylindrically symmetric gravitational field.

It is well-known [6] that the general static cylindrically-symmetric vacuum solution of Einstein equations is characterized by two free parameters. A simple representation of this is the so-called Kasner solution:

$$
d s^{2}=(k r)^{2 a} d t^{2}-(k r)^{2 c} d z^{2}-d r^{2}-\beta^{2}(k r)^{2(b-1)} r^{2} d \phi^{2}
$$

where $k$ sets the length scale, $\beta$ will be discussed below and $a, b, c$ satisfy the Kasner conditions:

$$
a+b+c=a^{2}+b^{2}+c^{2}=1 .
$$

A question of fundamental interest is the interpretation of these parameters and the connection between them and the internal properties of the matter distribution, i.e. of the cosmic string in the present context.

^Electronic address: verbin@oumail.openu.ac.il 
The simplest model for a cosmic string is the so called "gauge string" [2] which is an idealized cylindrical mass distribution with a finite radial extension having $\mathcal{T}_{0}^{0}=\mathcal{T}_{3}^{3}$ as the only non-vanishing components of the energy-momentum tensor. It is well-known by now that this string picks up from the Kasner family a very simple solution namely $a=c=0, b=1$ which is evidently locally flat. However, there is a global non-trivial effect i.e. the geometry is that of a cone. The parameter $\beta$ represents a conic angular deficit [07, 8] which is also related to the mass distribution of the source.

The explicit relation between the angular deficit $\delta \phi=2 \pi(1-\beta)$ and the "inertial mass" (per unit length) $\tilde{m}$, of a gauge string was found to be

$$
\delta \phi=8 \pi G \tilde{m}
$$

first [5] in the linearized approximation assuming also an infinitesimally thin source, and then [9, 10] by solving the full non-linear Einstein equations around a uniform source (constant $\mathcal{T}_{0}^{0}$ ) with a finite radius. The same relation was also derived [11] for a non-uniform source. Since the space-time around a gauge string is locally flat, this angular deficit is the only geometrical evidence of its existence.

Further study of the subject [12] involved a more realistic model (i.e. the abelian Higgs model) for the cosmic strings and the analysis of the full coupled field equations for the gravitational field and matter (scalar + vector) fields. It became clear that (1.3) is only an approximation and several corrections were calculated 13, 14, 15, 16.

The cosmic strings of the abelian Higgs model are the simplest and most studied ones. If however, gravity is a scalar-tensor theory as suggested by string theory, one should examine the existence of cosmic strings in this case as well. This has been done already for Brans-Dicke theory and its extensions [17, 18, 19] and for dilatonic gravity [20] where an extra scalar field (dilaton) couples to the Higgs system and to the metric tensor. Cosmic string solutions were found and their characteristics were discussed.

There exists yet another simple system which may give rise to cosmic strings where gravity is purely tensorial but couples non-minimally to the matter fields. It is the abelian Higgs model where the charged scalar field has a (non-minimal) "conformal" coupling to gravity. It seems that cosmic strings of this model have never been discussed in the literature, although it is not much more complicated than the minimally coupled abelian Higgs model. This paper contains therefore a verifications (by construction) of the existence of such solutions and a study of their characteristics. In order to have a basis for appreciating the new results, the next section (2) concentrates in cosmic strings in the minimally coupled case. The most important features are presented - most of them well-known but some interesting novelties are added. Section 3 contains the general analysis of the conformally coupled case and section 4 contains numerical results which establish the existence of the new kind of cosmic strings and a discussion of their main features. Generally, they are found to be similar to the minimally coupled ones.

\section{Abelian Higgs Cosmic Strings}

The action which allows self-gravitating abelian flux tubes is

$$
S=\int d^{4} x \sqrt{|g|}\left(\frac{1}{2} D_{\mu} \Phi^{*} D^{\mu} \Phi-\frac{\lambda}{4}\left(\Phi^{*} \Phi-v^{2}\right)^{2}-\frac{1}{4} F_{\mu \nu} F^{\mu \nu}+\frac{1}{16 \pi G} \mathcal{R}\right)
$$

where $\mathcal{R}$ is the Ricci scalar, $F_{\mu \nu}$ the abelian field strength, $\Phi$ is a complex scalar field and $D_{\mu}=$ $\nabla_{\mu}-i e A_{\mu}$ is the usual gauge covariant derivative. We use units in which $\hbar=c=1$.

Because of the cylindrical symmetry of the source we will use a line element of the form:

$$
d s^{2}=N^{2}(r) d t^{2}-d r^{2}-L^{2}(r) d \phi^{2}-K^{2}(r) d z^{2}
$$


and the usual Nielsen-Olesen ansatz for the +1 flux unit:

$$
\Phi=v f(r) e^{i \phi}, \quad A_{\mu} d x^{\mu}=\frac{1}{e}(1-P(r)) d \phi .
$$

This gives rise to the following field equations for the Abelian Higgs flux tube:

$$
\begin{aligned}
\frac{\left(N K L f^{\prime}\right)^{\prime}}{N K L}+\left(\lambda v^{2}\left(1-f^{2}\right)-\frac{P^{2}}{L^{2}}\right) f & =0 \\
\frac{L}{N K}\left(\frac{N K}{L} P^{\prime}\right)^{\prime}-e^{2} v^{2} f^{2} P & =0
\end{aligned}
$$

With the line element (2.2) the components of the Ricci tensor are:

$$
\mathcal{R}_{0}^{0}=-\frac{\left(L K N^{\prime}\right)^{\prime}}{N L K}, \quad \mathcal{R}_{r}^{r}=-\frac{N^{\prime \prime}}{N}-\frac{L^{\prime \prime}}{L}-\frac{K^{\prime \prime}}{K}, \quad \mathcal{R}_{\phi}^{\phi}=-\frac{\left(N K L^{\prime}\right)^{\prime}}{N L K}, \quad \mathcal{R}_{z}^{z}=-\frac{\left(N L K^{\prime}\right)^{\prime}}{N L K} .
$$

The source is described by the energy-momentum tensor with the following components:

$$
\begin{aligned}
& \mathcal{T}_{0}^{0}=\rho=\varepsilon_{s}+\varepsilon_{v}+\varepsilon_{s v}+u \\
& \mathcal{T}_{r}^{r}=-p_{r}=-\varepsilon_{s}-\varepsilon_{v}+\varepsilon_{s v}+u \\
& \mathcal{T}_{\phi}^{\phi}=-p_{\phi}=\varepsilon_{s}-\varepsilon_{v}-\varepsilon_{s v}+u \\
& \mathcal{T}_{z}^{z}=-p_{z}=\rho
\end{aligned}
$$

where

$$
\varepsilon_{s}=\frac{v^{2}}{2} f^{\prime 2}, \quad \varepsilon_{v}=\frac{P^{\prime 2}}{2 e^{2} L^{2}}, \quad \varepsilon_{s v}=\frac{v^{2} P^{2} f^{2}}{2 L^{2}}, \quad u=\frac{\lambda v^{4}}{4}\left(1-f^{2}\right)^{2} .
$$

For further use we define the magnetic field as:

$$
\mathcal{B}=-\frac{P^{\prime}}{e L}
$$

It turns out to be convenient to use Einstein equations in the form:

$$
\mathcal{R}_{\mu \nu}=-8 \pi G\left(\mathcal{T}_{\mu \nu}-\frac{1}{2} g_{\mu \nu} \mathcal{T}\right)
$$

where $\mathcal{T}$ is the contracted energy-momentum tensor: $\mathcal{T}=\rho-p_{z}-p_{r}-p_{\phi}$.

By insertion of $\mathcal{T}_{\mu \nu}$ one obtains:

$$
\begin{gathered}
\frac{\left(L K N^{\prime}\right)^{\prime}}{N L K}=4 \pi G\left(\rho+p_{r}+p_{\phi}+p_{z}\right)=8 \pi G\left(\varepsilon_{v}-u\right) \\
\frac{\left(N K L^{\prime}\right)^{\prime}}{N L K}=-4 \pi G\left(\rho-p_{r}+p_{\phi}-p_{z}\right)=-8 \pi G\left(\varepsilon_{v}+2 \varepsilon_{s v}+u\right) \\
\frac{\left(L N K^{\prime}\right)^{\prime}}{N L K}=-4 \pi G\left(\rho-p_{r}-p_{\phi}+p_{z}\right)=8 \pi G\left(\varepsilon_{v}-u\right)
\end{gathered}
$$

and instead of the "radial" part of (2.10) we take the following combination:

$$
\frac{N^{\prime}}{N} \frac{L^{\prime}}{L}+\frac{L^{\prime}}{L} \frac{K^{\prime}}{K}+\frac{K^{\prime}}{K} \frac{N^{\prime}}{N}=8 \pi G p_{r}=8 \pi G\left(\varepsilon_{s}+\varepsilon_{v}-\varepsilon_{s v}-u\right)
$$


which is not an independent equation but serves as a constraint. In vacuum the right-hand-sides of these equations vanish and the first three of them are trivially integrated. In this way we may get back Kasner's line element (1.1). This is therefore the asymptotic form of the metric tensor around any (transversally) localized source and especially around an abelian Higgs flux tube.

Moreover, it is easy to get convinced that due to the symmetry under boosts along the string axis, $K=N$.

The equations become more transparent if we express all lengths in terms of the scalar characteristic length scale $\left(1 / \sqrt{\lambda v^{2}}\right.$, the "correlation length" in the superconductivity terminology). We therefore change to the dimensionless length coordinate $x=\sqrt{\lambda v^{2}} r$ and metric component $\sqrt{\lambda v^{2}} L$ which we still denote by $L$. We also introduce the two parameters $\alpha=e^{2} / \lambda$ and $\gamma=8 \pi G v^{2}$. In terms of these new quantities we get a two parameter system of 4 coupled non-linear ordinary differential equations $\left(\right.$ Now of course $\left.{ }^{\prime}=d / d x\right)$ :

$$
\begin{gathered}
\frac{\left(N^{2} L f^{\prime}\right)^{\prime}}{N^{2} L}+\left(1-f^{2}-\frac{P^{2}}{L^{2}}\right) f=0 \\
\frac{L}{N^{2}}\left(\frac{N^{2} P^{\prime}}{L}\right)^{\prime}-\alpha f^{2} P=0 \\
\frac{\left(L N N^{\prime}\right)^{\prime}}{N^{2} L}=\gamma\left(\frac{P^{\prime 2}}{2 \alpha L^{2}}-\frac{1}{4}\left(1-f^{2}\right)^{2}\right) \\
\frac{\left(N^{2} L^{\prime}\right)^{\prime}}{N^{2} L}=-\gamma\left(\frac{P^{\prime 2}}{2 \alpha L^{2}}+\frac{P^{2} f^{2}}{L^{2}}+\frac{1}{4}\left(1-f^{2}\right)^{2}\right) .
\end{gathered}
$$

We have also to keep in mind the existence of the constraint (2.14) which gets the following form:

$$
\frac{N^{\prime}}{N}\left(2 \frac{L^{\prime}}{L}+\frac{N^{\prime}}{N}\right)=\gamma\left(\frac{f^{\prime 2}}{2}+\frac{P^{\prime 2}}{2 \alpha L^{2}}-\frac{P^{2} f^{2}}{2 L^{2}}-\frac{1}{4}\left(1-f^{2}\right)^{2}\right) .
$$

In order to get string solutions the scalar and gauge field should satisfy the following boundary conditions:

$$
\begin{aligned}
f(0) & =0, & & \lim _{x \rightarrow \infty} f(x)=1 \\
P(0) & =1, & & \lim _{x \rightarrow \infty} P(x)=0
\end{aligned}
$$

and regularity of the geometry on the symmetry axis will be guarantied by the "initial conditions":

$$
\begin{aligned}
L(0) & =0, & L^{\prime}(0) & =1 \\
N(0) & =1, & N^{\prime}(0) & =0
\end{aligned}
$$

It is well-known that even in flat space these field equations can only be solved numerically. However, much can be said about the nature of the solutions even without explicitly solving the field equations [12, 15, 21, 16]. The first is that the abelian Higgs string has a vanishing gravitational mass which means that the spacetime around the string is locally flat except in its core. However, there is a non-trivial global effect namely a conical structure of the space which is quantified by an angular deficit. This does not saturate all the possibilities. There are further types of solutions of (2.15)-(2.18) with the same boundary (and "initial") conditions which are not asymptotically flat but have interesting physical interpretation. Thus, a point in the $\alpha-\gamma$ plane does not always represent a 
single solution. The various solutions are distinguished by their asymptotic geometries. Here we will concentrate in the cosmic string solutions, i.e. those which are asymptotically conical.

For analyzing the solutions and obtaining the above-mentioned features and some additional ones, we introduce the Tolman mass (per unit length) of this system, $M$ :

$$
\begin{aligned}
G M & =2 \pi G \int_{0}^{\infty} d r N^{2} L\left(\rho+p_{r}+p_{\phi}+p_{z}\right)= \\
& =\frac{\gamma}{2} \int_{0}^{\infty} d x N^{2} L\left(\frac{P^{2}}{2 \alpha L^{2}}-\frac{1}{4}\left(1-f^{2}\right)^{2}\right)=\frac{1}{2} \lim _{x \rightarrow \infty}\left(L N N^{\prime}\right)
\end{aligned}
$$

where the last equality comes from the "temporal" Einstein equation.

The string angular deficit will be related to the "angular" Einstein equation by defining:

$$
\begin{aligned}
G W & =-2 \pi G \int_{0}^{\infty} d r N^{2} L\left(\rho-p_{r}+p_{\phi}-p_{z}\right)= \\
& =-\frac{\gamma}{2} \int_{0}^{\infty} d x N^{2} L\left(\frac{P^{\prime 2}}{2 \alpha L^{2}}+\frac{P^{2} f^{2}}{L^{2}}+\frac{1}{4}\left(1-f^{2}\right)^{2}\right)=\frac{1}{2}\left(\lim _{x \rightarrow \infty}\left(N^{2} L^{\prime}\right)-1\right)
\end{aligned}
$$

It is assumed of course that the integrals converge and the limits exist. This will be verified numerically later.

The asymptotic form of the metric tensor is easily found by direct integration of the two Einstein equations (2.17)-(2.18) using the boundary conditions and the definitions of $M$ and $W$. It is of the Kasner form

$$
N(x)=K(x) \sim \kappa x^{a}, \quad L(x) \sim \beta x^{b},
$$

with:

$$
a=\frac{2 G M}{2 G(2 M+W)+1}, \quad b=\frac{2 G W+1}{2 G(2 M+W)+1}, \quad \kappa^{2} \beta=2 G(2 M+W)+1 .
$$

The constant $\kappa$ appears free in the asymptotic solution, but it is uniquely fixed in the complete one by the boundary conditions on the metric.

Now we notice that the "radial" Einstein equation, (2.19) can be used at $x \rightarrow \infty$ to give in this case the following relation which is equivalent to the quadratic Kasner condition in (1.2):

$$
G M(G M+2 G W+1)=0 .
$$

This immediately indicates the existence of two branches of solutions. $M=0$ corresponds to the cosmic string branch since $a=c=0, b=1$. The other turns out to have non-flat asymptotic behavior ( $a=c=2 / 3, b=-1 / 3$ ), much like that of the Melvin solution and we may call it the "Melvin branch". We will not focus on this branch since it is clear by its asymptotics that it cannot represent cosmic strings. We will however refer to it on passing in order to complete the picture.

In the cosmic string branch one can easily identify the angular deficit $\delta \phi$ by:

$$
1-\frac{\delta \phi}{2 \pi}=L^{\prime}(\infty)=\frac{2 G W+1}{N^{2}(\infty)} .
$$

It is obvious that $W$ contains contribution not only from the inertial mass (integral over $\rho$ ), but also from the pressure terms so eq. (1.3), $\delta \phi=8 \pi G \tilde{m}$, is valid only in circumstances when the transverse pressures are negligible. Otherwise, one should replace $\tilde{m}$ by $\tilde{m}-\tilde{p}_{r}$, where $\tilde{p}_{r}$ is the appropriate integral over $p_{r}$ (with $N(\infty)$ absorbed in the measure). In short:

$$
\tilde{m}=2 \pi \int_{0}^{\infty} d r N^{2} L \rho / N^{2}(\infty), \quad \tilde{p}_{r}=2 \pi \int_{0}^{\infty} d r N^{2} L p_{r} / N^{2}(\infty)
$$


Recall also that $M=0$. Moreover, it is inconsistent to take $\kappa=N(\infty)=1$ since we have already made the choice $N(0)=1$ in order to be able to treat on the same footing the solutions in both branches. This factor represents a red/blue-shift of time between infinity and the core of the string and adds its own contribution to the angular deficit. We have therefore a generalized Vilenkin relation:

$$
\frac{\delta \phi}{2 \pi}=4 G\left(\tilde{m}-\tilde{p}_{r}\right)+1-\frac{1}{N^{2}(\infty)} .
$$

Actually this result does not depend upon the detailed properties of the model and is valid for any static matter distribution with cylindrical symmetry and boost symmetry along the string axis, provided it is localized enough so the relevant integrals converge. In the case of the minimally coupled abelian Higgs model one may get a more explicit expression by use of eqs. (2.7)-(2.8).

The angular deficit may be written in an alternative form if we start from the following identity:

$$
\frac{L^{\prime \prime}}{L}=\left(\frac{N^{\prime}}{N}\right)^{2}-4 \pi G\left(2 \rho+p_{r}+p_{\phi}\right)
$$

which is obtained for $\rho+p_{z}=0 \quad(\Rightarrow K=N)$ from (2.12) and (2.14). If we now multiply both sides by $L(r)$ and integrate we find after some manipulations (integrations by parts etc.) and use of $M=0$ that:

$$
\frac{\delta \phi}{2 \pi}=8 \pi G \int_{0}^{\infty} d r L\left(\rho+\frac{1}{4}\left(p_{r}+p_{\phi}\right)\right) .
$$

Note that the measure here is a purely two-dimensional one which is related to the induced metric of the constant time and constant $z$ surface. A "predecessor" of this relation was derived already by Garfinkle and Laguna [12, 15] although in a form where only the $\rho$ term of (2.31) was explicit and the second term was expressed in terms of the metric components. This result is also model-independent in the same sense as explained above. For the abelian Higgs model we obtain the following explicit expression:

$$
\frac{\delta \phi}{2 \pi}=4 \pi G \int_{0}^{\infty} d r L\left(2 \varepsilon_{s}+3 \varepsilon_{v}+2 \varepsilon_{s v}+u\right)
$$

An additional relation unnoticed so far is a simple connection between the angular deficit of an abelian Higgs string and the central value of the magnetic field $\mathcal{B}(0)$. One may prefer to use the dimensionless magnetic field $B(x)=-P^{\prime}(x) / L(x)=(8 \pi G / e) \alpha \mathcal{B} / \gamma$. At any rate the relation is:

$$
\frac{\delta \phi}{2 \pi}=1-\frac{1-\frac{\gamma}{\alpha} B(0)}{N^{2}(\infty)}=1-\frac{1-\frac{8 \pi G}{e} \mathcal{B}(0)}{N^{2}(\infty)} .
$$

This result is obtained from the following identity which amounts to an integration of one combination (i.e. the difference) of the two equations (2.17)-(2.18):

$$
\frac{d}{d x}\left[N^{2} L\left(\frac{N^{\prime}}{N}-\frac{L^{\prime}}{L}-\frac{\gamma}{\alpha} \frac{P P^{\prime}}{L^{2}}\right)\right]=0
$$

If we use this identity for the string branch at both $x \rightarrow 0$ and $x \rightarrow \infty$ we get the following which directly leads from 2.27) to (2.33):

$$
G W=-\frac{\gamma}{2 \alpha} B(0) .
$$

This relation is easily generalized for the Melvin branch:

$$
G(M-W)=\frac{\gamma}{2 \alpha} B(0) .
$$


Since now $M \neq 0$ we may use the relation (2.26) to solve for the mass and the $W$-parameter in terms of the single combination $\gamma B(0) / \alpha$ :

$$
\begin{array}{r}
G M=\frac{\gamma}{3 \alpha} B(0)-\frac{1}{3} \\
G W=-\frac{\gamma}{6 \alpha} B(0)-\frac{1}{3} .
\end{array}
$$

Note that in the case of the -1 flux unit the magnetic field changes sign so $B(0)$ should actually be replaced in the above equations by $|B(0)|$ in order to cover both cases.

As numerical analysis shows (sec. 4), the angular deficit increases with $\gamma$ and decreases with $\alpha$. An analytic demonstration for the $\gamma$ dependence is obtained in the simplest solution for this system, namely the Bogomolnyi solution [22, 23] which exists for $\alpha=2$. In this case the system is in a state where the repulsion due to vector exchange is exactly balanced by the attraction due to the scalar exchange. This is reflected by an identically vanishing gravitational potential, namely, $N(x)=1$. The field equations turn out to be equivalent to the following first order equations:

$$
\begin{aligned}
L f^{\prime}-P f & =0 \\
P^{\prime}+L\left(1-f^{2}\right) & =0 \\
L^{\prime}-\frac{\gamma}{2} P\left(1-f^{2}\right) & =1-\frac{\gamma}{2} .
\end{aligned}
$$

Using e.g. (2.27) and (2.35) with $B(0)=1$ (which follows from (2.40), we find a simple expression for the angular deficit which was already noticed by Linet [22]:

$$
\delta \phi=\pi \gamma
$$

This linear increase is evidently associated with a maximal value of $\gamma=2$. Beyond this value the solutions are closed, namely, outside the core of the string, $L(x)$ decreases linearly and vanishes at a finite distance from the string axis. The geometry is still conic but of an inverted one where the apex of the cone is at the point where $L=0$ [9, 24].

If we now return to arbitrary values of $\alpha$ and $\gamma$ and look back at (2.33) we find a critical value for the central magnetic field of an abelian Higgs cosmic string:

$$
\mathcal{B}_{c}=e / 8 \pi G, \quad B_{c}=\alpha / \gamma
$$

Note that for a given $e$, say the electromagnetic value, the value of the critical magnetic field, $\mathcal{B}_{c}$ is unique. However, the dimensionless critical magnetic field is given in terms of both $\alpha$ and $\gamma$ so there is a whole curve in the $\alpha-\gamma$ plane which corresponds to solutions with the same critical asymptotic behavior.

\section{Abelian Higgs Cosmic Strings with Non-Minimal Coupling to Gravity}

The possibility of non-minimal coupling $\left(R \Phi^{2}\right)$ between gravity and scalar fields was investigated by many authors and the special coefficient of $1 / 12$ was found to be of special interest due to the conformal symmetry associated with such a coupling [25, 26, 27, 28]. It is however quite surprising that the possibility of cosmic strings in this system has never been discussed in the literature. The action contains thus an additional term for the non-minimal "conformal coupling":

$$
S=\int d^{4} x \sqrt{|g|}\left(\frac{1}{2} D_{\mu} \Phi^{*} D^{\mu} \Phi-\frac{\lambda}{4}\left(|\Phi|^{2}-v^{2}\right)^{2}-\frac{1}{12} \mathcal{R}|\Phi|^{2}-\frac{1}{4} F_{\mu \nu} F^{\mu \nu}+\frac{1}{16 \pi G} \mathcal{R}\right) .
$$


The modification in the abelian Higgs sector is only a "renormalization" of the self interaction coupling constant $\lambda$ to $(1-\gamma / 6) \lambda$ which results from the identity

$$
\mathcal{R}=8 \pi G \mathcal{T}=8 \pi G v^{2} \lambda\left(v^{2}-|\Phi|^{2}\right)
$$

On the other hand there are considerable modifications in the Einstein equations due to the new energy-momentum tensor:

$$
\mathcal{T}_{\mu \nu}=\mathcal{T}_{\mu \nu}^{(\text {minimal })}+\frac{1}{6}\left(g_{\mu \nu} \nabla^{\rho} \nabla \rho|\Phi|^{2}-\nabla_{\mu} \nabla_{\nu}|\Phi|^{2}-\mathcal{G}_{\mu \nu}|\Phi|^{2}\right)
$$

or by using Einstein equation:

$$
\mathcal{T}_{\mu \nu}=\frac{1}{1-\frac{\gamma}{6}|\Phi|^{2}}\left[\mathcal{T}_{\mu \nu}^{(\text {minimal })}+\frac{1}{6}\left(g_{\mu \nu} \nabla^{\rho} \nabla_{\rho}|\Phi|^{2}-\nabla_{\mu} \nabla_{\nu}|\Phi|^{2}\right)\right]
$$

$\mathcal{T}_{\mu \nu}^{(\text {minimal })}$ is of course the energy-momentum tensor of the minimally coupled system of the previous section.

The same cosmic string ansatz gives now the following form of Einstein equations:

$$
\begin{aligned}
& \frac{\left(L K N^{\prime}\right)^{\prime}}{N L K}=\frac{8 \pi G}{1-\frac{\gamma}{6} f^{2}}\left(\frac{1}{3} \varepsilon_{s}+\varepsilon_{v}+\frac{1}{3} \varepsilon_{s v}-w+\frac{v^{2}}{3} \frac{N^{\prime}}{N} f f^{\prime}\right) \\
& \frac{\left(N K L^{\prime}\right)^{\prime}}{N L K}=\frac{8 \pi G}{1-\frac{\gamma}{6} f^{2}}\left(\frac{1}{3} \varepsilon_{s}-\varepsilon_{v}-\frac{5}{3} \varepsilon_{s v}-w+\frac{v^{2}}{3} \frac{L^{\prime}}{L} f f^{\prime}\right) \\
& \frac{\left(L N K^{\prime}\right)^{\prime}}{N L K}=\frac{8 \pi G}{1-\frac{\gamma}{6} f^{2}}\left(\frac{1}{3} \varepsilon_{s}+\varepsilon_{v}+\frac{1}{3} \varepsilon_{s v}-w+\frac{v^{2}}{3} \frac{K^{\prime}}{K} f f^{\prime}\right)
\end{aligned}
$$

where we use the field equation for the scalar and define the combination:

$$
w=\frac{\lambda v^{4}}{4}\left(1-f^{2}\right)\left(1-\frac{1}{3}\left(1+\frac{\gamma}{3}\right) f^{2}\right)
$$

The radial constraint is now:

$$
\frac{N^{\prime}}{N} \frac{L^{\prime}}{L}+\frac{L^{\prime}}{L} \frac{K^{\prime}}{K}+\frac{K^{\prime}}{K} \frac{N^{\prime}}{N}=\frac{8 \pi G}{1-\frac{\gamma}{6} f^{2}}\left(\varepsilon_{s}+\varepsilon_{v}-\varepsilon_{s v}-u+\frac{v^{2}}{3} \frac{(N K L)^{\prime}}{N K L} f f^{\prime}\right) .
$$

A glance at the Einstein equations for this case reveals that their structure is quite similar to that for minimal coupling. The main difference is the appearance of a "field dependent gravitational constant", $G /\left(1-\frac{\gamma}{6} f^{2}\right)$ because of which we may expect more pronounced gravitational effects, since it is larger than $G$. Moreover, there seems to be a critical value of the scalar vacuum expectation value $v$ since $\gamma=6$ is singular as seen for example from the "renormalization" of $\lambda$ and from other results that will follow. We will thus concentrate in the region $\gamma<6$.

By subtracting (3.7) from (3.5) one can show that the boost symmetry along the string axis is still valid hence $K=N$. We also note that the last term in the right-hand side in each of (3.5)-(3.7) may be transferred to the left-hand-side to form a total derivative so we write:

$$
\left(\left(1-\frac{\gamma}{6} f^{2}\right) L N N^{\prime}\right)^{\prime}=8 \pi G N^{2} L\left(\frac{1}{3} \varepsilon_{s}+\varepsilon_{v}+\frac{1}{3} \varepsilon_{s v}-w\right)
$$




$$
\left(\left(1-\frac{\gamma}{6} f^{2}\right) N^{2} L^{\prime}\right)^{\prime}=8 \pi G N^{2} L\left(\frac{1}{3} \varepsilon_{s}-\varepsilon_{v}-\frac{5}{3} \varepsilon_{s v}-w\right)
$$

It is also useful to write the equations explicitly in terms of the dimensionless variables:

$$
\begin{aligned}
& \left(\left(1-\frac{\gamma}{6} f^{2}\right) L N N^{\prime}\right)^{\prime}=\gamma N^{2} L\left(\frac{f^{\prime 2}}{6}+\frac{P^{\prime 2}}{2 \alpha L^{2}}+\frac{P^{2} f^{2}}{6 L^{2}}-\frac{1}{4}\left(1-f^{2}\right)\left(1-\frac{1}{3}\left(1+\frac{\gamma}{3}\right) f^{2}\right)\right) \\
& \left(\left(1-\frac{\gamma}{6} f^{2}\right) N^{2} L^{\prime}\right)^{\prime}=\gamma N^{2} L\left(\frac{f^{\prime 2}}{6}-\frac{P^{\prime 2}}{2 \alpha L^{2}}-\frac{5}{6} \frac{P^{2} f^{2}}{L^{2}}-\frac{1}{4}\left(1-f^{2}\right)\left(1-\frac{1}{3}\left(1+\frac{\gamma}{3}\right) f^{2}\right)\right) .
\end{aligned}
$$

A first order equation analogous to (2.34) is obtained from the difference (3.12)-(3.13):

$$
\frac{d}{d x}\left[N^{2} L\left(\left(1-\frac{\gamma}{6} f^{2}\right)\left(\frac{N^{\prime}}{N}-\frac{L^{\prime}}{L}\right)-\frac{\gamma}{\alpha} \frac{P P^{\prime}}{L^{2}}\right)\right]=0
$$

The constraint (3.9) gets the form

$$
\frac{N^{\prime}}{N}\left(2 \frac{L^{\prime}}{L}+\frac{N^{\prime}}{N}\right)=\frac{\gamma}{1-\frac{\gamma}{6} f^{2}}\left(\frac{f^{\prime 2}}{2}+\frac{P^{\prime 2}}{2 \alpha L^{2}}-\frac{P^{2} f^{2}}{2 L^{2}}-\frac{1}{4}\left(1-f^{2}\right)^{2}+\frac{1}{3} \frac{\left(N^{2} L\right)^{\prime}}{N^{2} L} f f^{\prime}\right) .
$$

Integration of $(3.12$ ) and $(3.13)$ gives the following integral expressions for $M$ and $W$ :

$$
\begin{aligned}
G M & =\frac{1}{2} \lim _{x \rightarrow \infty}\left(L N N^{\prime}\right)= \\
& =\frac{\gamma}{2\left(1-\frac{\gamma}{6}\right)} \int_{0}^{\infty} d x N^{2} L\left(\frac{f^{\prime 2}}{6}+\frac{P^{\prime 2}}{2 \alpha L^{2}}+\frac{P^{2} f^{2}}{6 L^{2}}-\frac{1}{4}\left(1-f^{2}\right)\left(1-\frac{1}{3}\left(1+\frac{\gamma}{3}\right) f^{2}\right)\right) \\
G W & =\frac{1}{2}\left(\lim _{x \rightarrow \infty}\left(N^{2} L^{\prime}\right)-1\right)= \\
& =\frac{\gamma}{2\left(1-\frac{\gamma}{6}\right)} \int_{0}^{\infty} d x N^{2} L\left(\frac{f^{\prime 2}}{6}-\frac{P^{\prime 2}}{2 \alpha L^{2}}-\frac{5}{6} \frac{P^{2} f^{2}}{L^{2}}-\frac{1}{4}\left(1-f^{2}\right)\left(1-\frac{1}{3}\left(1+\frac{\gamma}{3}\right) f^{2}\right)\right) .(3.1
\end{aligned}
$$

The asymptotic form of the metric tensor is still described by 2.24)-(2.26) and the angular deficit is still given by (2.27) or (2.29) provided we use the appropriate energy-momentum tensor. Since (2.31) is also still valid, an expression analogous to 2.32 may be immediately found:

$$
\frac{\delta \phi}{2 \pi}=4 \pi G \int_{0}^{\infty} d r \frac{L}{1-\frac{\gamma}{6} f^{2}}\left(\varepsilon_{s}+3 \varepsilon_{v}+\varepsilon_{s v}+\frac{\lambda v^{4}}{4}\left(1-f^{2}\right)\left(1+\left(1-\frac{\gamma}{3}\right) f^{2}\right)+v^{2} \frac{N^{\prime}}{N} f f^{\prime}\right)
$$

A relation between the angular deficit and the magnetic field exists here as well. We can use eq. (3.14) to obtain:

$$
\left(1-\frac{\gamma}{6}\right) G(M-W)=\frac{\gamma}{2 \alpha} B(0)-\frac{\gamma}{12} .
$$

From here we find that for the cosmic string branch

$$
G W=-\frac{\frac{\gamma}{2 \alpha} B(0)-\frac{\gamma}{12}}{1-\frac{\gamma}{6}}
$$

and

$$
\frac{\delta \phi}{2 \pi}=1-\frac{1-\frac{\gamma}{\alpha} B(0)}{\left(1-\frac{\gamma}{6}\right) N^{2}(\infty)}
$$


while for the Melvin branch we get $M$ and $W$ in terms of the central magnetic field:

$$
\begin{array}{r}
G M=\frac{\frac{\gamma}{\alpha} B(0)-1}{3\left(1-\frac{\gamma}{6}\right)} \\
G W=-\frac{\frac{\gamma}{2 \alpha} B(0)+1-\frac{\gamma}{4}}{3\left(1-\frac{\gamma}{6}\right)} .
\end{array}
$$

Note that although the relation between the central magnetic field and the angular deficit is modified, the critical magnetic field is still given by the minimal coupling expression, (2.43).

We thus expect to find in the case of conformal coupling, cosmic string solutions that do not differ significantly from the minimally coupled ones. This will indeed be the main conclusion but there is one important exception: the Bogomolnyi cosmic strings. As mentioned above, this is the case where there is a complete balance between the repulsive and attractive contributions (to $\mathcal{T}_{\mu \nu}$ ) which is reflected by the fact that $N(x)=1$ is a consistent solution. The straightforward way to study the analogous situation for conformal coupling is to examine whether the system still admits $N(x)=1$ as a solution. It turns out that this is not the case, and $N(x)=1$ is inconsistent with the field equations. One may arrive at this conclusion by a direct computation of the derivatives of $N(x)$ at $x=0$ up to order $p$ (exploiting all the field equations) for any $\alpha$ and $\gamma$ and studying the possibility that they all vanish simultaneously. It is found that $p$ cannot be greater than 9 , namely, there cannot exist a solution where all the first 10 derivatives of $N(x)$ at $x=0$ vanish. This evidently excludes the possibility of a constant $N(x)$. Since high order derivatives are involved, the algebraic manipulations are rather cumbersome and they will not be shown here explicitly.

Further study of the nature of the solutions is possible only by numerical analysis. The results are described in the next section.

\section{Numerical Results and Conclusion}

In this section the essential characteristics of the cosmic strings with conformal coupling to gravity are presented, mainly in a graphical manner. For comparison, the corresponding characteristics for minimal coupling are also shown. The results were obtained by the MATHEMATICA package using an iterative procedure for solving the four coupled field equations. In order to insure the consistency of the results several checks were made using the constraints and relations like (3.15) and (3.19) and their minimal coupling analogues.

The scalar and gauge field look generally as in flat space. The gauge field, or $P(x)$ is sensitive to $\alpha$ but does not depend very much on $\gamma$. It slightly concentrates toward the axis when $\gamma$ is increased. Fig.11 depicts $P(x)$ for $\alpha=0.25,0.5,1,2,4$. For each value of $\alpha, \gamma$ takes values in steps of 0.25 starting from $\gamma=0$ (flat space flux tube) up to a maximal value which depends on $\alpha: 1,1,1.25,1.75,2$ for the above values respectively. Altogether there are 33 points in the $\alpha-\gamma$ plane represented in the figure. The fact that the vector field concentrates while $\alpha$ increases, results from the fact that $\alpha$ represents the ratio between the scalar and the vector characteristic length scales. One should however keep in mind that the behavior with respect to "real" distance, $r=x / \sqrt{\lambda v^{2}}$ may be different since changes in $\alpha$ and $\gamma$ are associated with changes in $\lambda$ and $v$ and an increase of those tends to expand $P(r)$.

The scalar field is quite insensitive to changes in both $\alpha$ and $\gamma$ as can be learnt from Fig.2: An increase of $\alpha$ and decrease of $\gamma$ have a similar weak effect of increasing the rate of approach of $f(x)$ towards 1. Equivalently, the slope of the scalar field on the axis increases with $\alpha$ and decreases with $\gamma$. The $f(x)$ curves correspond to the same 33 points in the $\alpha-\gamma$ plane mentioned above.

Fig. 3 presents the behavior of the magnetic field. For the sake of clarity only the extreme values of $\alpha$ and $\gamma$ appear. The general tendency is that the magnetic field increases with both $\alpha$ and $\gamma$. However, this tendency reverses for large $x$ since $B(x)$ (as well as the other fields) approaches faster 
its vacuum value for larger values of $\alpha$. There is also a slight decrease of the magnetic field on the axis in the minimally coupled string for $\alpha<2$.

The metric components are more sensitive to changes in $\alpha$ and $\gamma$ and there is a boundary in the $\alpha-\gamma$ plane beyond which no open solutions exist at all. A complete mapping of the $\alpha-\gamma$ plane is a matter for further analysis. A general idea as of the shape of this boundary (i.e. along which $\delta \phi=2 \pi$ ) may be obtained however from Fig. 10.

The conformal coupling indirectly intensifies the gravitational effects with respect to the case of minimal coupling. The temporal metric component $N(x)$ changes its behavior significantly; especially for $\alpha<2$ where $N(x)$ is a decreasing function for all $\gamma$ in the minimally coupled case but not so for conformal coupling. For $\alpha \geq 2$, where the general form of $N(x)$ is similar for both cases, the red/blue-shift factor $N(\infty)$ is much larger for conformal coupling than for minimal coupling. These features are evident from Fig. 4 Fig. 6 which present $N(x)$ for several representative points in the $\alpha-\gamma$ plane.

The most prominent feature of the cosmic string solutions is the angular deficit $\delta \phi$ which is in one-to-one correspondence with the asymptotic value of the slope of the angular metric component $L(x)$. Fig. 7 -Fig. 8 show this function and short inspection shows that $\delta \phi$ decreases with $\alpha$ and increases with $\gamma$ in both types of coupling. The angular deficit is always larger in the conformally coupled case.

The $\alpha$ and $\gamma$ dependence of these two characteristics $N(\infty)$ and $\delta \phi$ is more clearly seen in Fig.9 and Fig.10 which show a portion of the $\alpha-\gamma$ plane. Note the very different behavior of the red/blue-shift factor $N(\infty)$ in both cases. For minimal coupling the line $\alpha=2$ is the Bogomolnyi line which separates between $N(\infty)<1$ and $N(\infty)>1$. A similar $N(\infty)=1$ line exists for the conformal coupling; however as the non-existence of Bogomolnyi strings implies, it does not correspond to solutions with $N(x)=1$ as happens for minimal coupling, but merely to the case of no red/blue-shift between infinity and the core of the string.

the abelian Higgs cosmic strings with conformal coupling seem therefore to be as respectable as their minimally coupled counterparts. Both types share the same general tendencies and typical characteristics and most of the investigation may be done for both cases along parallel paths. The only significant difference is the non-existence of the Bogomolnyi cosmic strings in the case of conformal coupling.

\section{Acknowledgements}

I am grateful to R. Brustein, A. Davidson, A.L. Larsen, R. Madden and N.K. Nielsen for useful discussions and correspondence. 


\section{References}

[1] A.Vilenkin and E.P.S. Shellard, Cosmic strings and other Topological Defects (Cambridge Univ. Press, Cambridge, 1994).

[2] T.W.B Kibble and M. Hindmarsh, Rep. Progr. Phys. 58, 477 (1995).

[3] T.W.B Kibble, J. Phys. A9, 1387 (1976).

[4] Ya. B. Zel'dovich, Mon. Not. R. Astron. Soc. 192, 663 (1980).

[5] A. Vilenkin, Phys. Rev. D23, 852 (1981).

[6] D. Kramer, H. Stephani, E. Herlt and M. MacCallum, Exact Solutions of Einstein's Field Equations (Cambridge Univ. Press, Cambridge, England 1980).

[7] L. Marder, Proc. Roy. Soc. London A 252, 45 (1959).

[8] W.B. Bonnor, J. Phys. A 12, 847 (1979).

[9] J.R. Gott, Astrophys. J. 288, 422 (1985).

[10] W.A. Hiscock, Phys. Rev. D31, 3288 (1985).

[11] B. Linet, Gen. Relativ. Gravit. 17, 1109 (1985).

[12] D. Garfinkle, Phys. Rev. D32, 1323 (1985).

[13] P. Laguna-Castillo and R.A. Matzner, Phys. Rev.D36, 3663 (1987).

[14] D. Garfinkle and P. Laguna, Phys. Rev. D39, 1552 (1989).

[15] P. Laguna and D. Garfinkle, Phys. Rev. D40, 1011 (1989).

[16] J. Colding, N.K. Nielsen and Y. Verbin, Phys. Rev. D56, 3371 (1997).

[17] C.Gundlach and M.Ortiz, Phys. Rev. D42, 2521 (1990).

[18] L.O.Pimental and A.N.Morales, Rev. Mex. Fis.36 S199 (1990).

[19] M.E.X.Guimaraes, Class. Quantum Grav. 14435 (1997).

[20] R. Gregory and C. Santos, Phys. Rev. D56, 1194 (1997).

[21] V.P. Frolov, W. Israel and W.G. Unruh, Phys Rev. D39, 1084 (1989).

[22] B. Linet, Phys. Lett. A 124, 240 (1987).

[23] A. Comtet and G.W. Gibbons, Nucl. Phys. B299, 719 (1988).

[24] M.E. Ortiz, Phys. Rev. D43, 2521 (1991).

[25] R. Penrose, Proc. Roy. Soc. London A 284, 159 (1965).

[26] C.G. Callan, S. Coleman and R. Jackiw, Ann. Phys. (N.Y.) 59, 42 (1970).

[27] J. D. Bekenstein, Ann. Phys. (N.Y.) 82, 535 (1974).

[28] N.D. Birrell and P.C.W. Davies, Quantum Fields in Curved Space (Cambridge Univ. Press, Cambridge, England 1984). 

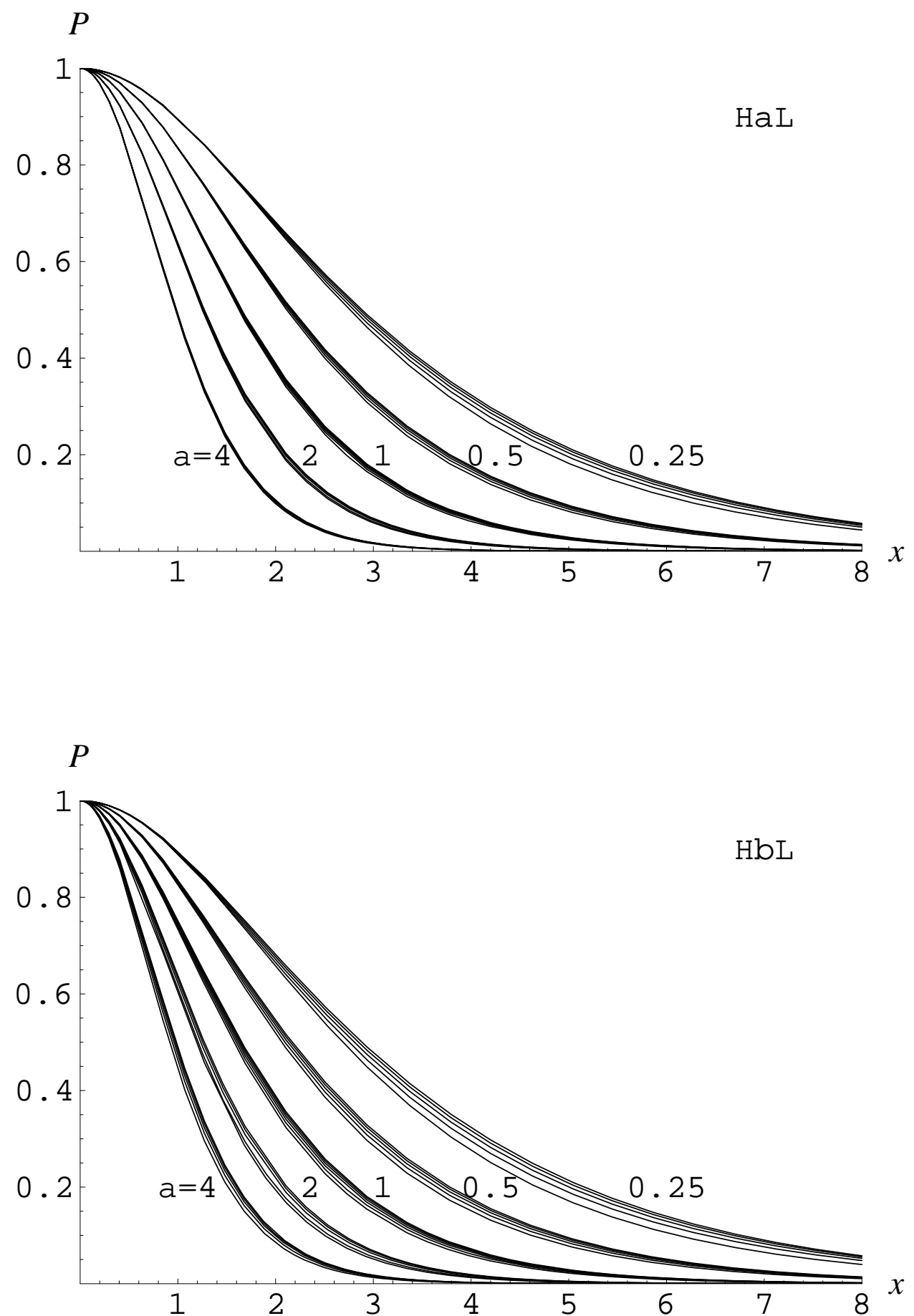

Figure 1: The magnitude of the gauge field $P(x)$ for $\alpha=0.25,0.5,1,2,4$ and several values of $\gamma$ - see text. (a) minimal coupling. (b) conformal coupling. 

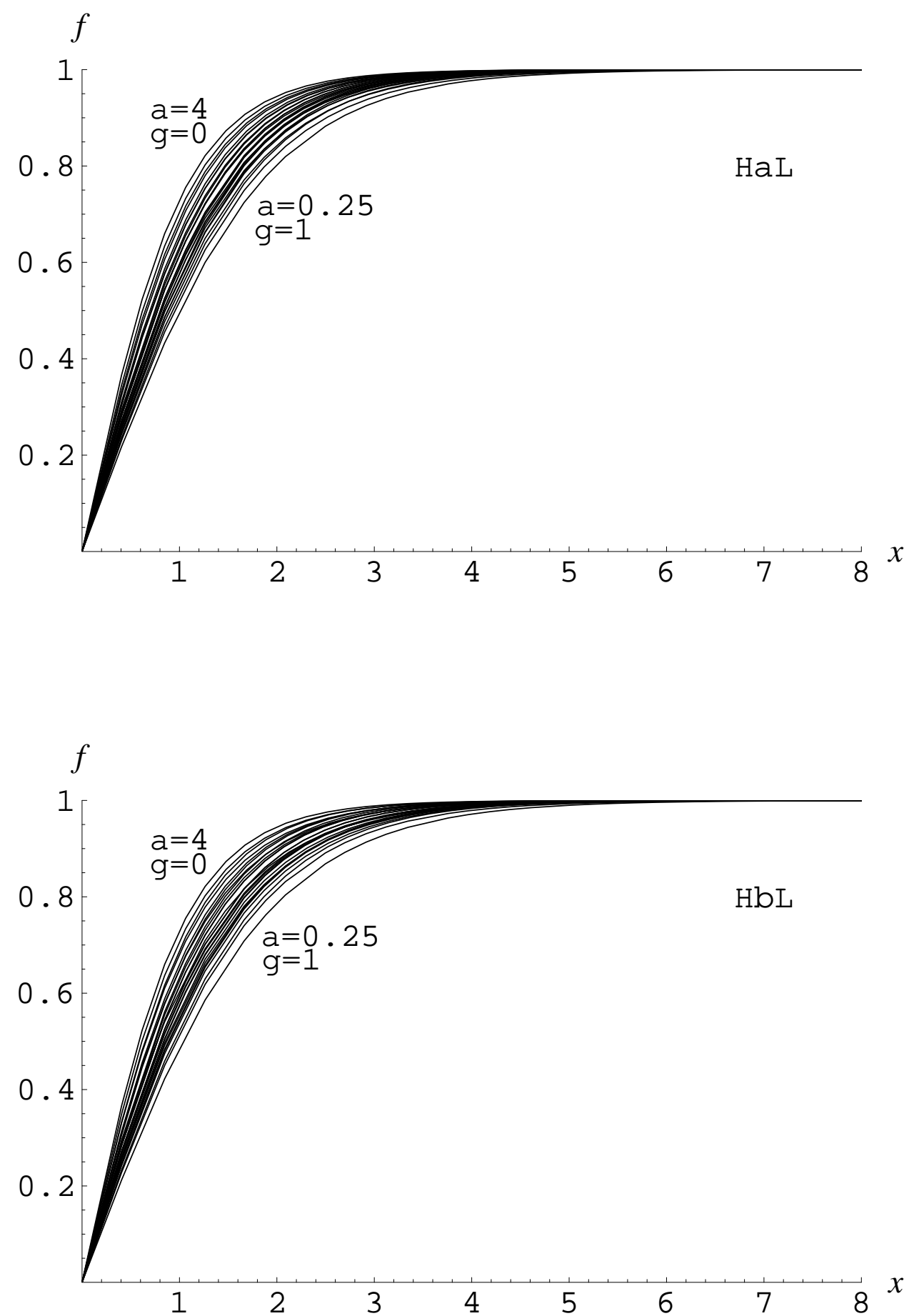

Figure 2: The magnitude of the scalar field $f(x)$ for $\alpha=0.25,0.5,1,2,4$ and several values of $\gamma$ - see text. The "envelope" corresponds to the values shown. (a) minimal coupling. (b) conformal coupling. 

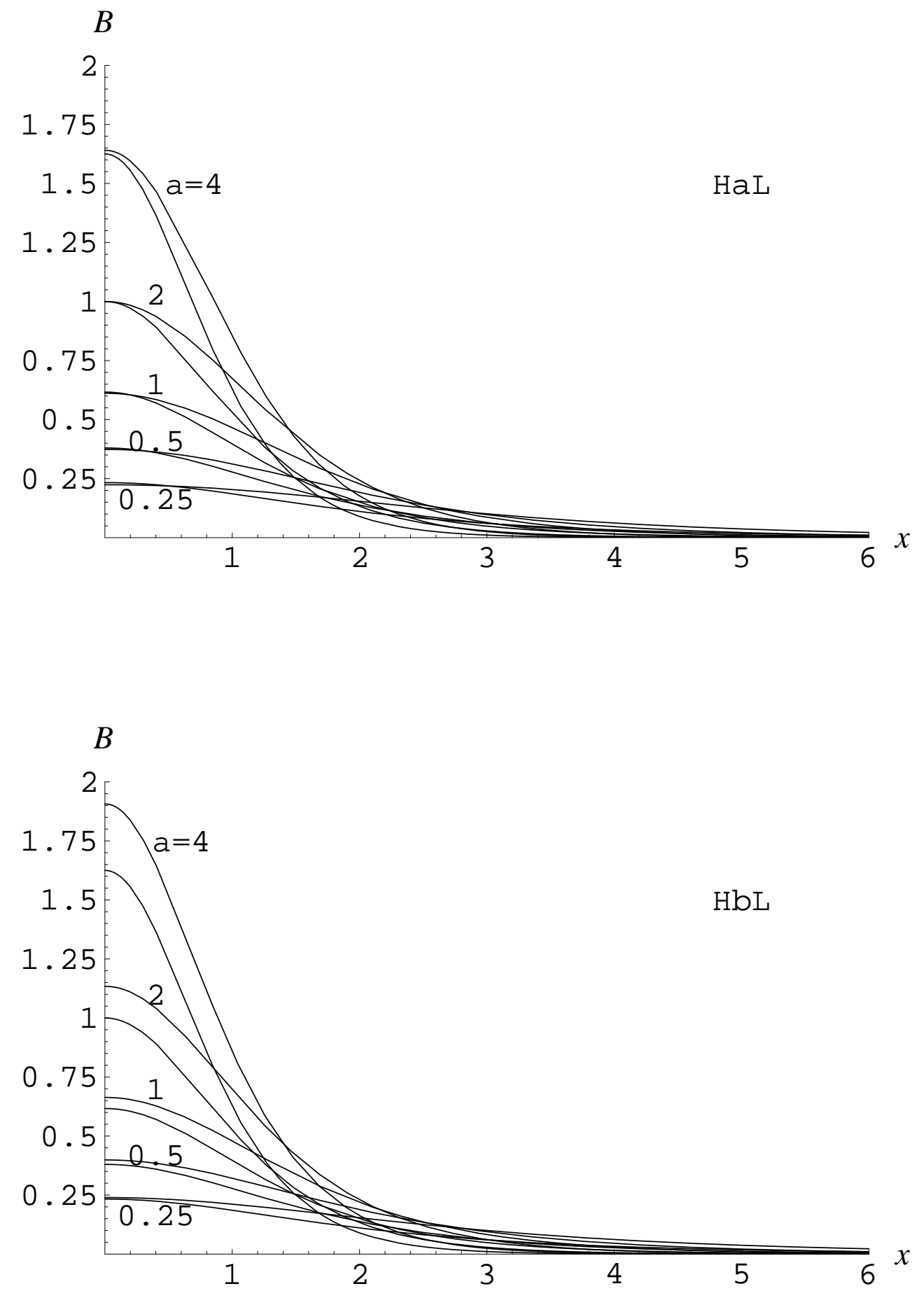

Figure 3: The magnitude of the magnetic field $B(x)$ for $\alpha=0.25,0.5,1,2,4$. Two curves are shown for each value of $\alpha: \gamma=0$ (the lower) and respectively $\gamma=1,1,1.25,1.75,2$. (a) minimal coupling. (b) conformal coupling. 

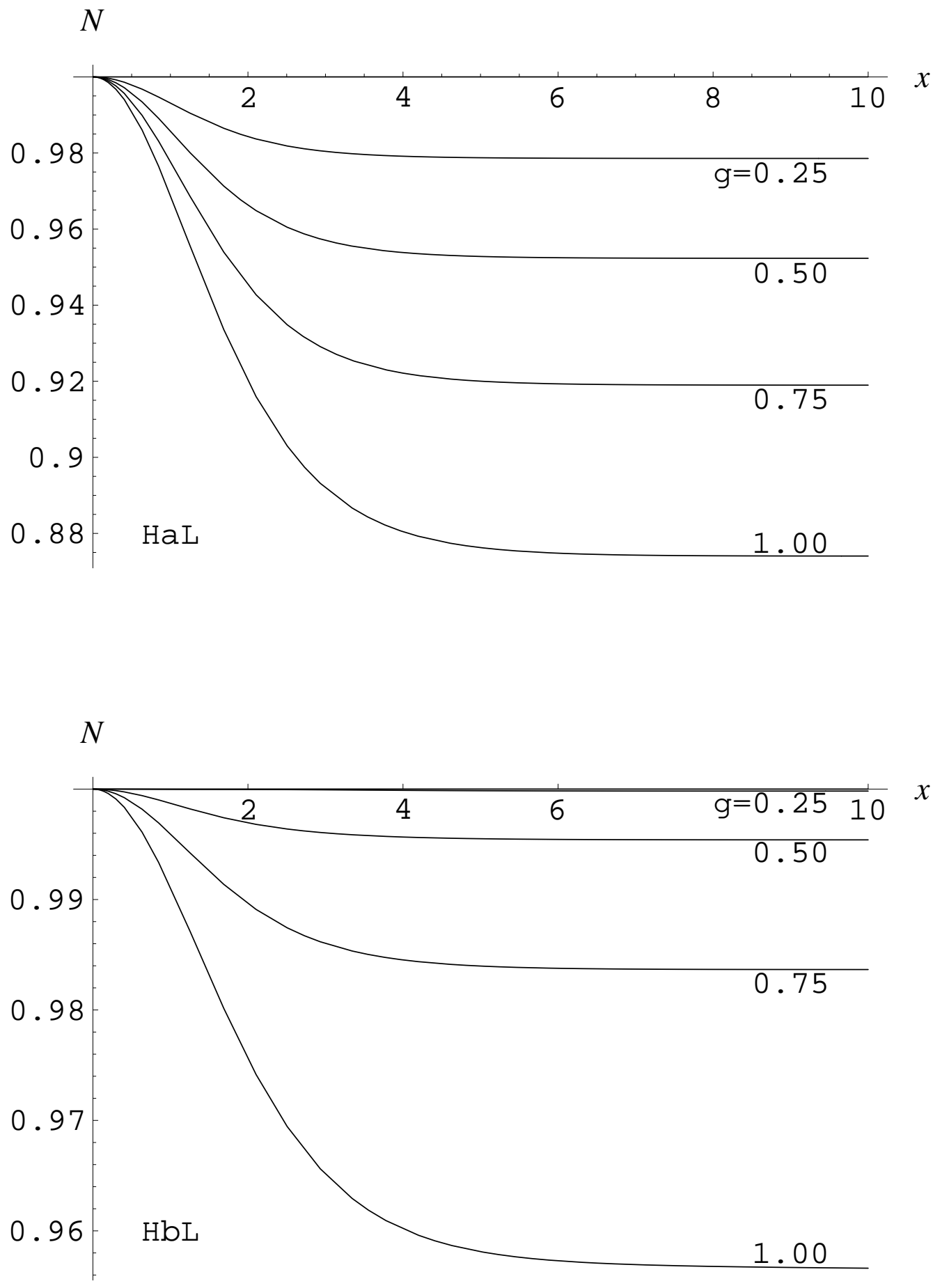

Figure 4: The magnitude of the metric component $N(x)$ for $\alpha=0.25$ and $\gamma=0,0.25,0.50,0.75,1.00$.

(a) minimal coupling. (b) conformal coupling. Note the difference in scale. $\gamma=0$ corresponds to $N(x)=1$; for $\gamma=0.25$ the deviation with respect to $N(x)=1$ is beyond the resolution of the graph. 

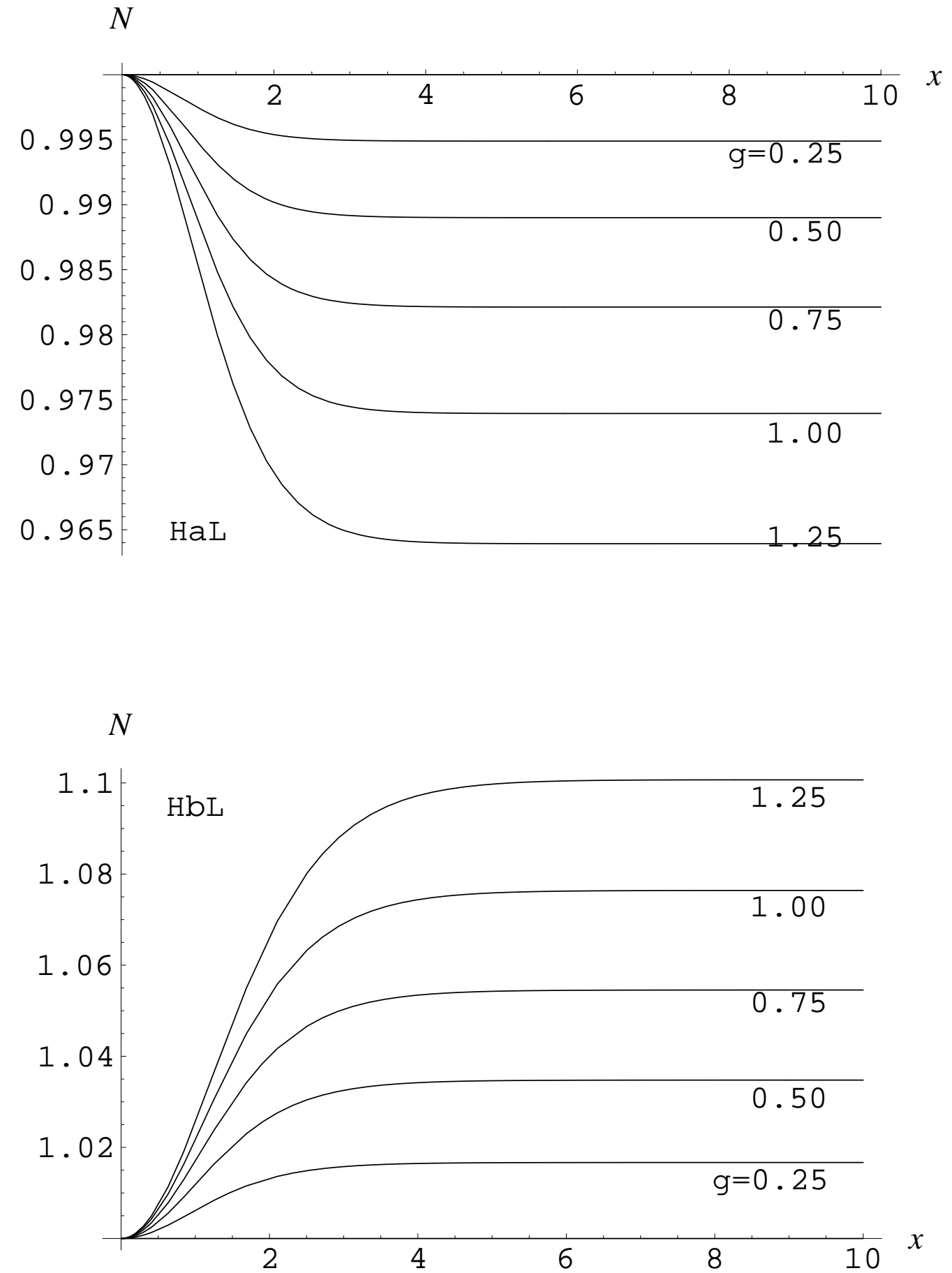

Figure 5: The magnitude of the metric component $N(x)$ for $\alpha=1$ and $\gamma=0,0.25,0.50,0.75,1.00,1.25$.

(a) minimal coupling. (b) conformal coupling. Note the difference in scale. $\gamma=0$ corresponds to $N(x)=1$. 

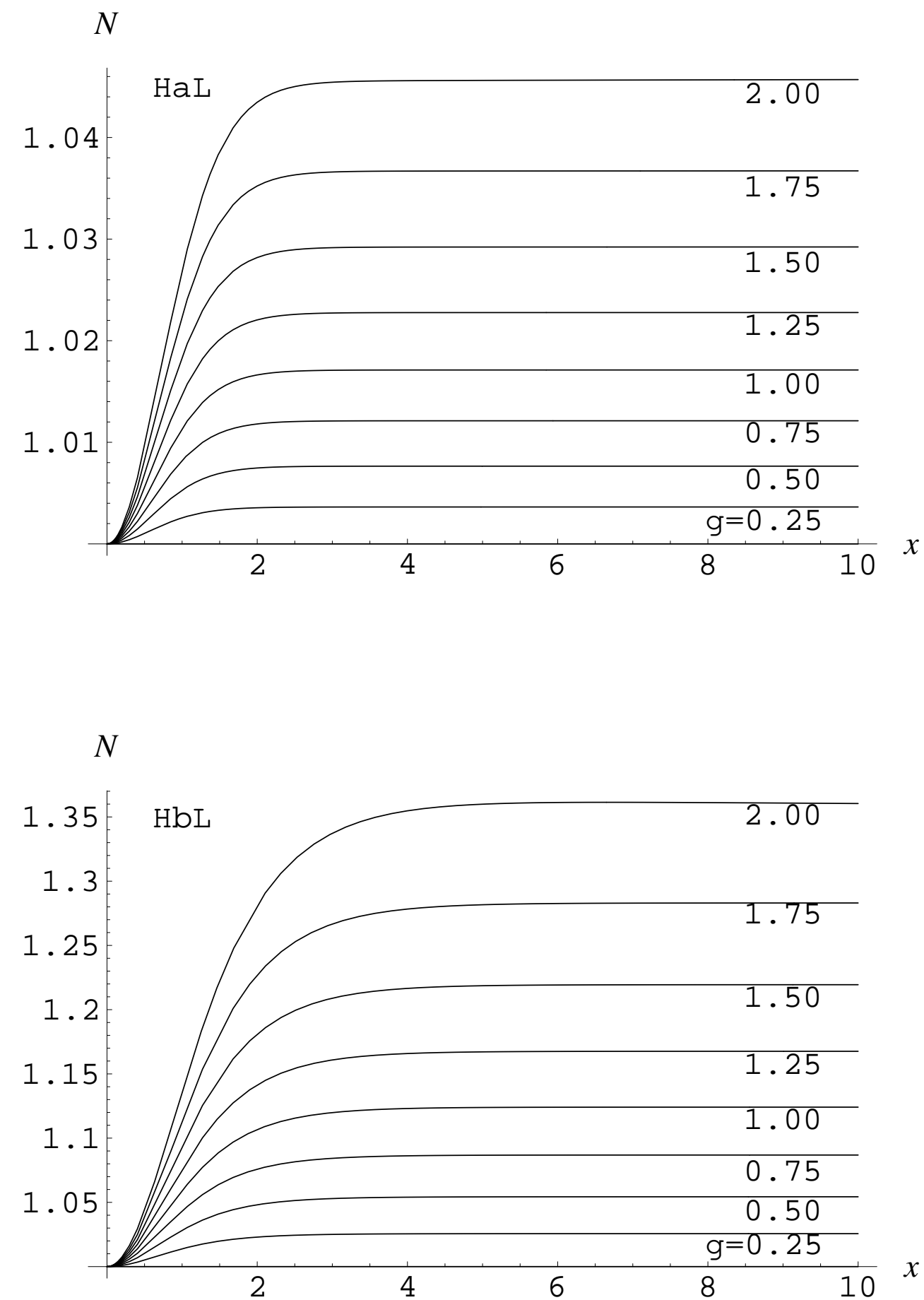

Figure 6: The magnitude of the metric component $N(x)$ for $\alpha=4$ and $\gamma=0,0.25,0.50,0.75,1.00,1.25,1.50$, 1.75, 2.00. (a) minimal coupling. (b) conformal coupling. Note the difference in scale. $\gamma=0$ corresponds to $N(x)=1$. 

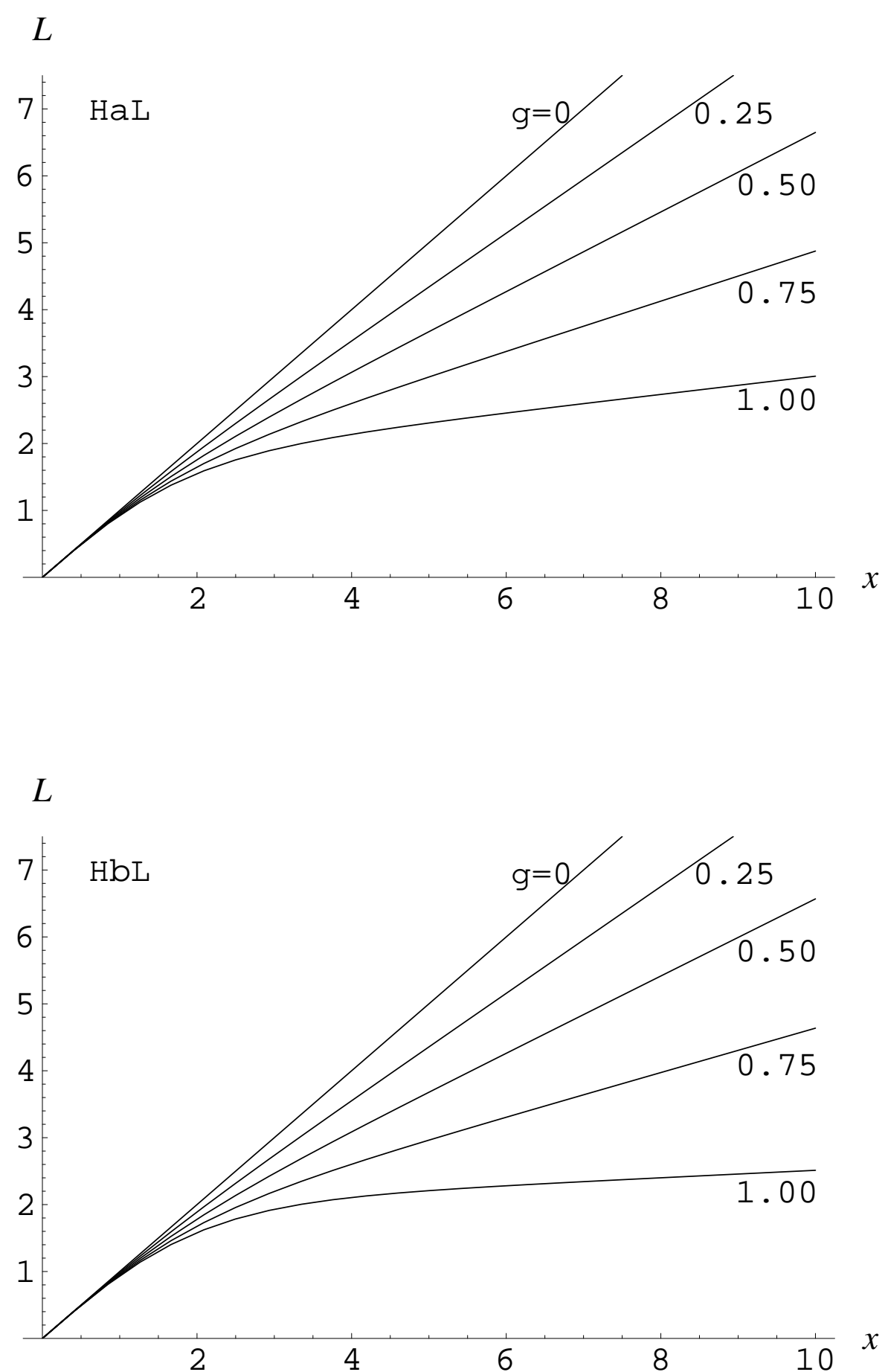

Figure 7: The magnitude of the metric component $L(x)$ for $\alpha=0.25$ and $\gamma=0,0.25,0.50,0.75,1.00$. (a) minimal coupling. (b) conformal coupling. 

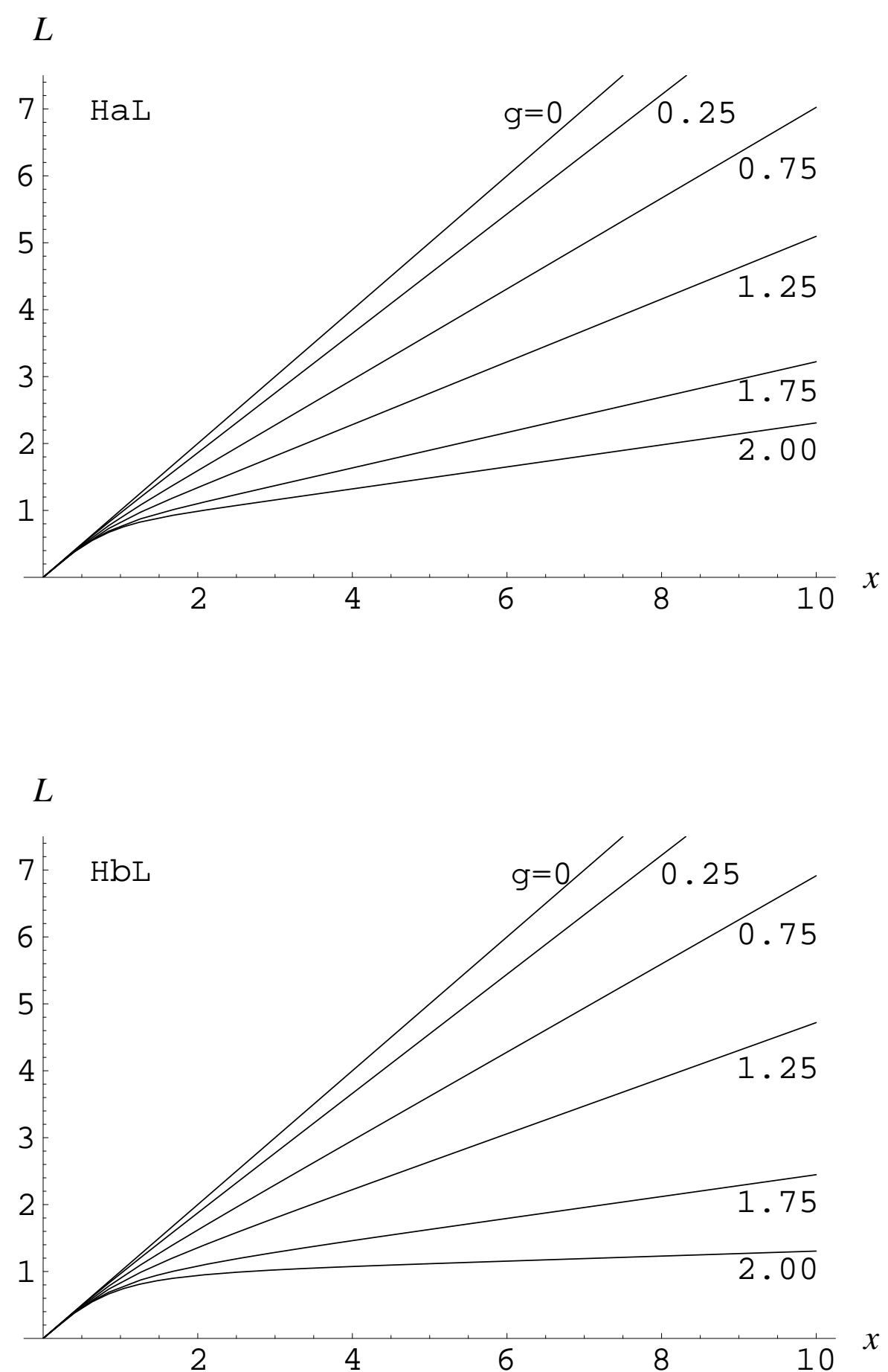

Figure 8: The magnitude of the metric component $L(x)$ for $\alpha=4$ and $\gamma=0,0.25,0.75,1.25,1.75,2.00$. (a) minimal coupling. (b) conformal coupling. 

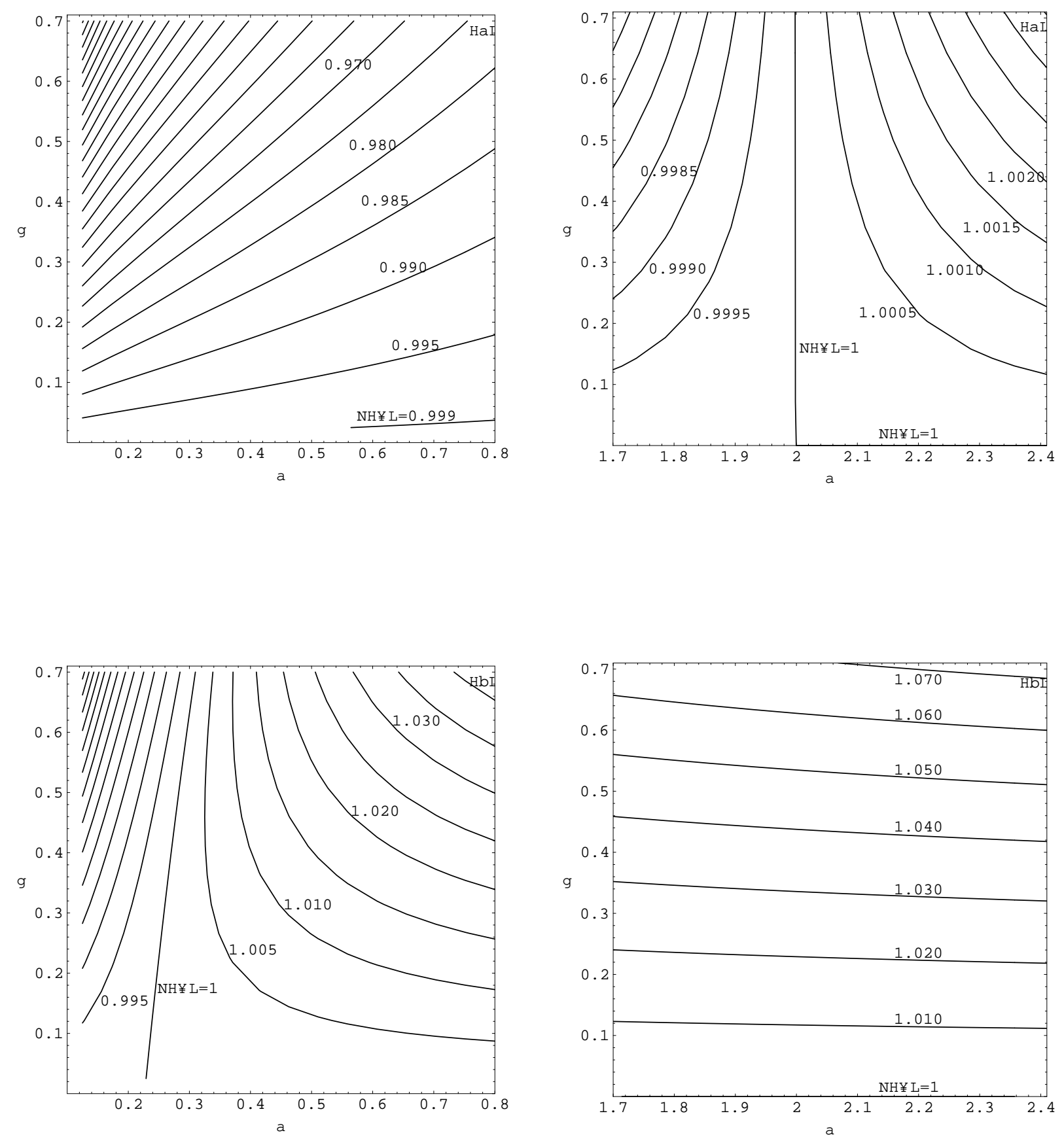

Figure 9: Curves of constant red/blue-shift factor $N(\infty)$ in two portions of the $\alpha$ - $\gamma$ plane. Note the non-equal spacing of the curves. (a) minimal coupling. (b) conformal coupling. 

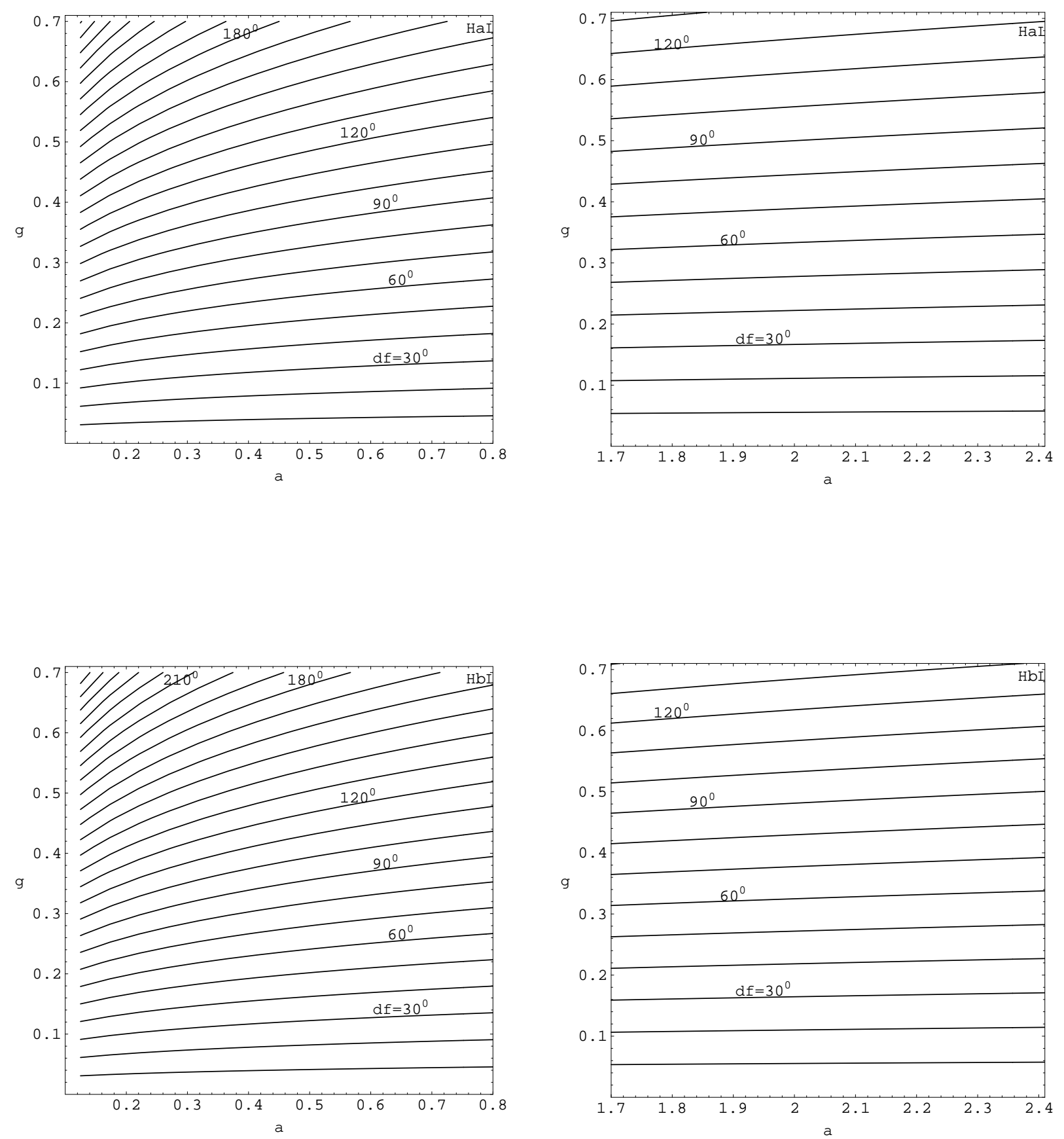

Figure 10: Curves of constant angular deficit $\delta \phi$ in two portions of the $\alpha-\gamma$ plane. The curves are spaced in steps of $10^{0}$. (a) minimal coupling. (b) conformal coupling. 